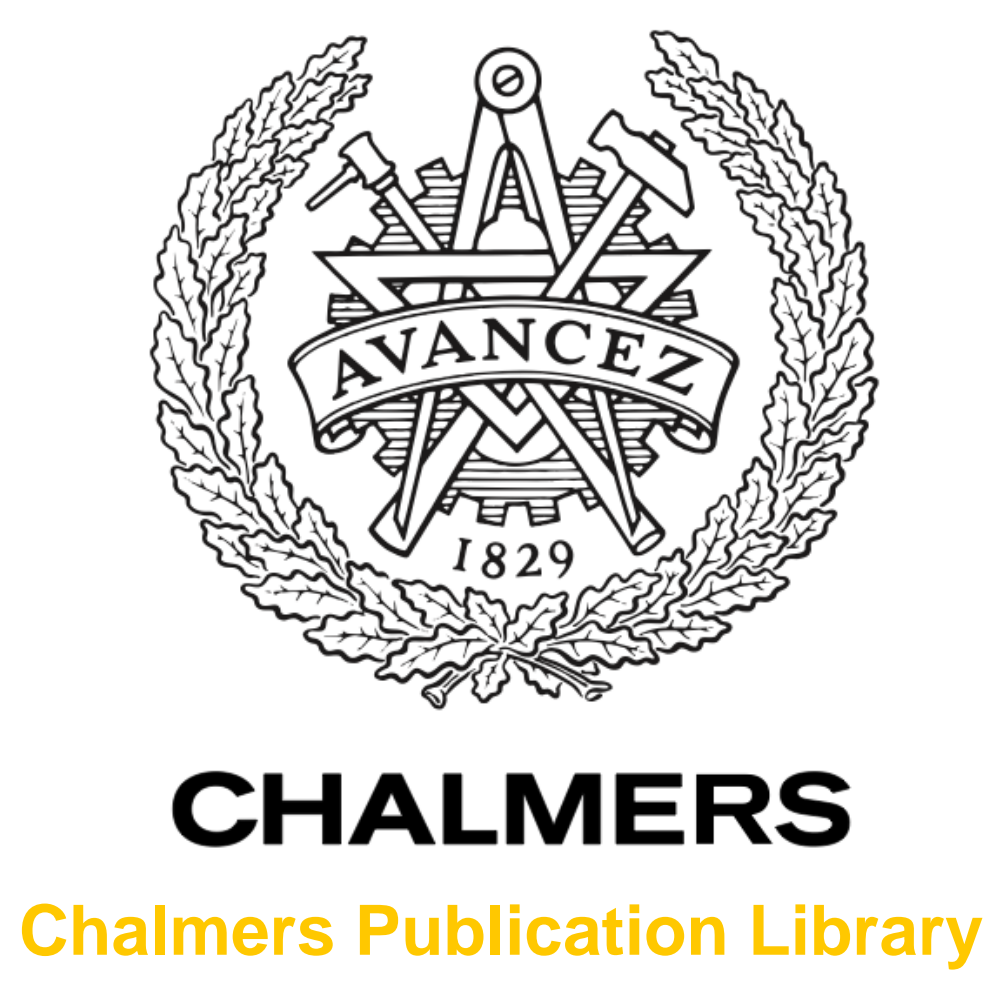

\title{
Enhanced Vehicle Positioning in Cooperative ITS by Joint Sensing of Passive Features
}

This document has been downloaded from Chalmers Publication Library (CPL). It is the author's version of a work that was accepted for publication in:

\section{IEEE International Conference on Intelligent Transportation Systems}

Citation for the published paper:

Soatti, G. ; Nicoli, M. ; Garcia, N. et al. (2017) "Enhanced Vehicle Positioning in

Cooperative ITS by Joint Sensing of Passive Features". IEEE International Conference on

Intelligent Transportation Systems

Downloaded from: http://publications.lib.chalmers.se/publication/252735

Notice: Changes introduced as a result of publishing processes such as copy-editing and formatting may not be reflected in this document. For a definitive version of this work, please refer to the published source. Please note that access to the published version might require a subscription. 


\title{
Enhanced Vehicle Positioning in Cooperative ITS by Joint Sensing of Passive Features
}

\author{
Gloria Soatti*, Monica Nicoli*, Nil Garcia ${ }^{\dagger}$, Benoit Denis ${ }^{\ddagger}$, Ronald Raulefs ${ }^{\S}$ and Henk Wymeersch ${ }^{\dagger}$ \\ *Politecnico di Milano, Milan, Italy \\ ${ }^{\dagger}$ Chalmers University of Technology, Gothenburg, Sweden

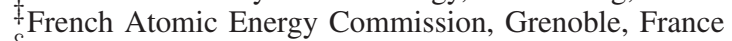 \\ $\S_{\text {German Aerospace Center (DLR), Wessling, Germany }}$ \\ Email:\{gloria.soatti,monica.nicoli\}@polimi.it,\{nilg,henkw\}@chalmers.se,benoit.denis@cea.fr,ronald.raulefs@dlr.de
}

\begin{abstract}
Satellite-based navigation systems, such as Global Positioning System (GPS) or Galileo, are the most common and accessible techniques for vehicle positioning. However, in dense urban areas, even if combined with vehicle on-board sensors, they lead to large localization errors due to multipath and signal blockage. In recent years, cooperative intelligent transportation systems (C-ITS) have gained increasing attention as they allow vehicles to cooperate and broadcast safety-related information to the neighbors through vehicle-to-vehicle $(\mathrm{V} 2 \mathrm{~V})$ communications. In this paper, a novel cooperative positioning method is developed by exploiting $\mathrm{V} 2 \mathrm{~V}$ communications without using explicit V2V ranging. Vehicles localize, in a distributed way, a set of jointly sensed non-cooperative features (e.g., people, traffic lights) and use them as common noisy reference points to implicitly enhance their own position accuracy. Distributed belief propagation is combined with consensus-based estimation of the features' positions to enable cooperative localization of vehicles. Numerical results show that the proposed method is able to significantly enhance the GPS-based vehicle location accuracy, reducing the error by approximately a factor 2 in the considered scenario, or even more in setups with denser feature deployments.
\end{abstract}

\section{INTRODUCTION}

Accurate position information is an essential component in Intelligent Transportation Systems (ITS), such as navigation, assisted driving and road safety [1]. Global Positioning System (GPS) enables to easily obtain information on vehicle position with accuracy of 5-10 meters under favorable conditions [2], but performances are degraded in harsh environments such as urban canyons and tunnels. Moreover, stand-alone GPS does not meet the requirements of safety-critical ITS services [3].

Recently, dedicated short range communication (DSRC) standards [4], relying on IEEE 802.11p technology, have been developed to support Vehicle-to-Vehicle (V2V) and/or Vehicleto-Infrastructure (V2I) communications in Cooperative ITS (C-ITS). These technologies can be exploited to achieve a more accurate and reliable vehicle localization through cooperative positioning (CP) [5,6]. In this context, extensive studies have been carried out to combine GPS measurements with other independent information, such as digital road maps, reference landmarks and/or inter-vehicle ranging measurements [7]-[9]. In particular, [7] proposes a CP algorithm, where a signal-strength-based ranging technique, integrated with road map information and vehicle kinematics, is used to improve position accuracy. Another CP method is proposed in [8], where GPS-based location information and high-resolution

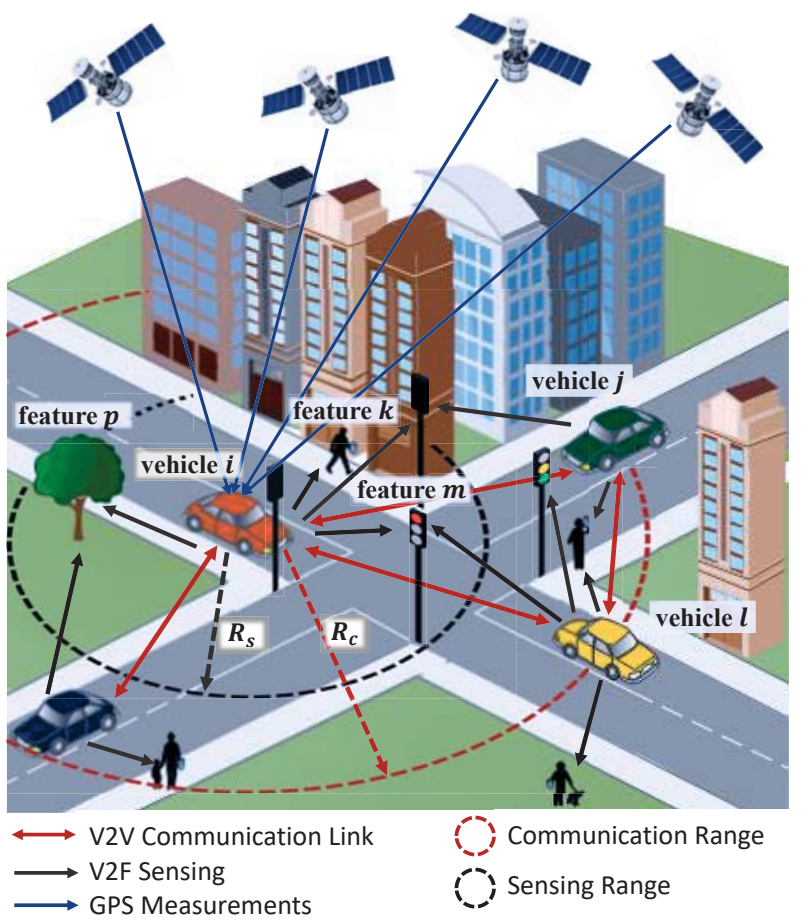

Fig. 1. Vehicular network with cooperative vehicles and non-cooperative features (i.e., people, traffic lights and trees).

maps are exchanged amongst vehicles to augment positioning performance. Though these solutions have shown to enhance location accuracy, they either rely on complex techniques with high computational cost, or require particular hardware or adhoc infrastructures, which make them hard to deploy. Furthermore, such methods use explicit $\mathrm{V} 2 \mathrm{~V}$ ranging measurements which tend to be of low quality (e.g., power observations), are not available in current standards for vehicular communications (e.g., time-based range measurements in IEEE 802.11p), or require specific hardware that may not be available (e.g., UWB radios) [9].

In this paper, we propose a novel implicit cooperative positioning (ICP) algorithm to improve the GPS-based vehicle location accuracy by interaction amongst vehicles through $\mathrm{V} 2 \mathrm{~V}$ communication links, as exemplified in Fig. 1. Differently from existing $\mathrm{CP}$ methods based on $\mathrm{V} 2 \mathrm{~V}$ ranging, a set of non-cooperative features (e.g., people, traffic lights or 

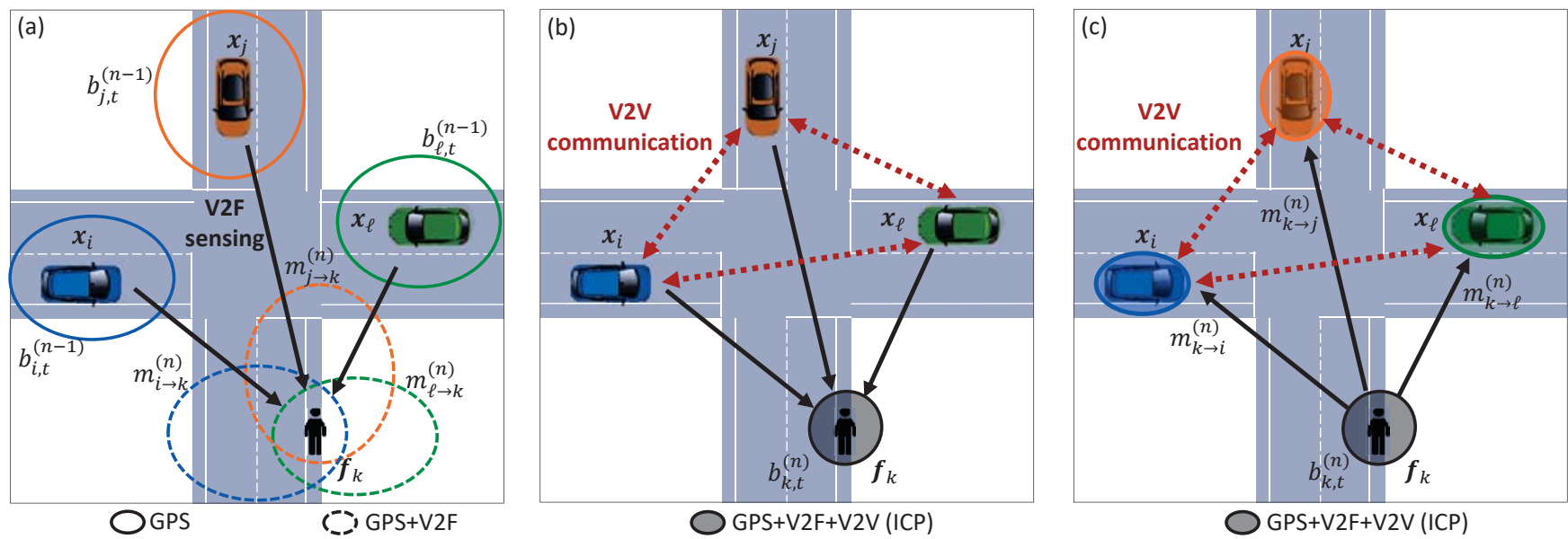

Fig. 2. Example of ICP procedure with three vehicles and one feature. (a) Vehicle/feature position information, represented as Gaussian beliefs (solid/dashed colored ellipses), obtained from on-board GPS and relative V2F sensing system. (b) Distributed fusion of vehicles' data over V2V links for cooperative localization of the feature (black ellipse). (c) Implicit improvement of the vehicle location information (colored ellipse) based on the refined feature localization.

trees) is passively detected and jointly localized by the vehicles so as to create a network of additional reference stations for GPS augmentation. As features are passive objects, vehicles have only some noisy information about the vehicle-to-feature (V2F) relative locations made by their on-board systems, such as radar or camera. In contrast to conventional map-based localization, the proposed method does not require an offline map, though it can be used if available. To take advantage of this online dynamic information, we develop a consensusbased belief propagation (BP) algorithm for solving the vehicle positioning problem.

The proposed approach is exemplified in Fig. 2, where three vehicles cooperatively localize a passive feature by sharing over V2V links their own information about the feature position based on on-board GPS and radar systems. Location information is modelled as a Gaussian belief, as shown in Fig. 2-(a) by the 1- $\sigma$ elliptical contours around vehicles and features. Combination of multi-vehicle data allows each vehicle to obtain a more accurate estimate of the feature position (Fig. 2-(b)), which is then used to enhance the vehicles' location accuracy itself (Fig. 2-(c)). The procedure, iterated till convergence, is implemented in a fully distributed way by combining a BP message passing scheme (for beliefs' exchange among vehicles) with a consensus approach (for distributed estimation of feature positions). Performance analysis in a simulated urban scenario shows that the proposed ICP method provides significant gain in terms of location accuracy with respect to conventional GPS positioning.

\section{Problem Formulation}

We consider a network of $N_{v}$ cooperating vehicles as in Fig. 1. The position of vehicle $i=1, \ldots, N_{v}$, denoted as $\mathbf{x}_{i, t}=\left[x_{x i, t}, x_{y i, t}\right]^{\mathrm{T}} \in \mathbb{R}^{2 \times 1}$, evolves over time $t$ according to the dynamic model [7]:

$$
\mathbf{x}_{i, t}=\mathbf{x}_{i, t-1}+T_{s} \mathbf{v}_{i, t-1}+T_{s} \mathbf{w}_{i, t-1},
$$

where $\mathbf{v}_{i, t-1}=\left[\begin{array}{ll}v_{x i, t} & v_{y i, t}\end{array}\right]^{\mathrm{T}} \in \mathbb{R}^{2 \times 1}$ is the vehicle velocity measured by the inner speedometer at the time $t-1, T_{s}$ is the sampling interval and $\mathbf{w}_{i, t-1} \sim \mathcal{N}\left(\mathbf{0}, \mathbf{Q}_{i, t-1}\right)$ is the zeromean Gaussian driving process.

The vehicular network is modelled as a time-varying undirected graph, $\mathcal{G}_{t}=\left(\mathcal{V}, \mathcal{E}_{t}\right)$, with vertices $\mathcal{V}=\left\{1, \ldots, N_{v}\right\}$ representing the vehicles and edges $\mathcal{E}_{t}$ the $\mathrm{V} 2 \mathrm{~V}$ communication links. Assuming that each vehicle has a communication range $R_{c}$ (see the dotted red circle in Fig. 1), the edge set is $\mathcal{E}_{t}=\left\{(i, j) \in \mathcal{V} \times \mathcal{V}:\left\|\mathbf{x}_{i, t}-\mathbf{x}_{j, t}\right\| \leq R_{c}\right\}$, with vehicles $i$ and $j$ connected if and only if their distance is lower or equal to $R_{c}$ (red links in Fig. 1) and with $\|\cdot\|$ being the Frobenius norm. The set of neighbors that directly communicates with vehicle $i \in \mathcal{V}$ is denoted as $\mathcal{J}_{i, t}=\left\{j \in \mathcal{V}:(i, j) \in \mathcal{E}_{t}\right\}$, its cardinality, or vehicle degree, as $d_{i, t}=\left|\mathcal{J}_{i, t}\right|$ and the maximum degree as $\Delta_{t}=\max d_{i, t}$.

As shown in Fig. 1, the scenario involves also a set $\mathcal{F}=\left\{1, \ldots, N_{f}\right\}$ of static passive features (e.g., people, traffic lights or trees), which are non-cooperative entities sensed by the vehicle's on-board equipment (e.g., radar or camerabased detector) and used as common noisy reference points for cooperative localization. Note that the method can be easily generalized to moving features. At time $t=1$, no prior knowledge on the feature positions $\mathbf{f}_{k}=\left[f_{x k}, f_{y k}\right]^{\mathrm{T}} \in$ $\mathbb{R}^{2 \times 1}, \forall k \in \mathcal{F}$ is assumed to be available. Due to the limited sensing range $R_{s}$, each vehicle $i$ senses only a subset of all features $\mathcal{F}_{i, t}=\left\{k \in \mathcal{F}:\left\|\mathbf{f}_{k}-\mathbf{x}_{i, t}\right\| \leq R_{s}\right\} \subseteq \mathcal{F}$ (dotted black circle in Fig. 1).

At time $t$, two types of location measurements are available at each vehicle: the GPS fix and the relative V2F position with respect to all features within the sensing range. The GPS measurement of vehicle $i$ location is modelled as:

$$
\mathbf{y}_{i, t}^{(\mathrm{GPS})}=\mathbf{x}_{i, t}+\mathbf{n}_{i, t}^{(\mathrm{GPS})}
$$

with $\mathbf{n}_{i, t}^{(\mathrm{GPS})} \sim \mathcal{N}\left(\mathbf{0}, \mathbf{R}_{i, t}^{(\mathrm{GPS})}\right)$ being the GPS error [7] [10]. The $\mathrm{V} 2 \mathrm{~F}$ relative location observation $\mathbf{y}_{i, k, t}^{(\mathrm{V} 2 \mathrm{~F})} \in \mathbb{R}^{2 \times 1}$ made by vehicle- $i$ on feature $k \in \mathcal{F}_{i, t}$ is given by:

$$
\mathbf{y}_{i, k, t}^{(\mathrm{V} 2 \mathrm{~F})}=q\left(\mathbf{f}_{k}-\mathbf{x}_{i, t}\right)+\mathbf{n}_{i, k, t}^{(\mathrm{V} 2 \mathrm{~F})}=q\left(\boldsymbol{\delta}_{i, k, t}\right)+\mathbf{n}_{i, k, t}^{(\mathrm{V} 2 \mathrm{~F})},
$$


where $\mathbf{n}_{i, k, t}^{(\mathrm{V} 2 \mathrm{~F})} \sim \mathcal{N}\left(\mathbf{0}, \mathbf{R}_{i, k, t}^{(\mathrm{V} 2 \mathrm{~F})}\right)$ is the measurement uncertainty and the deterministic function $q\left(\boldsymbol{\delta}_{i, k, t}\right)$ models the relation to the V2F relative position $\boldsymbol{\delta}_{i, k, t}=\mathbf{f}_{k}-\mathbf{x}_{i, t}$. Depending on the class of on-board sensors detecting the feature, the measurement may represent the V2F range $q\left(\boldsymbol{\delta}_{i, k, t}\right)=$ $\left\|\boldsymbol{\delta}_{i, k, t}\right\|$, the angle $q\left(\boldsymbol{\delta}_{i, k, t}\right)=\angle\left(\boldsymbol{\delta}_{i, k, t}\right)$, or the relative position $q\left(\boldsymbol{\delta}_{i, k, t}\right)=\boldsymbol{\delta}_{i, k, t}$. In this study, we focus on the latest case assuming that both range and angle measurements are available from on-board vehicle radar equipment. However, the framework is general enough to include other classes of measurements. The measurement errors $\mathbf{n}_{i, t}^{(\mathrm{GPS})}$ and $\mathbf{n}_{i, k, t}^{(\mathrm{V} 2 \mathrm{~F})}$ are assumed to be mutually independent and also independent over vehicles, features and time. We assume perfect association among measurements and features at each vehicle.

The problem investigated in this paper is the estimation of the position $\mathbf{x}_{i, t}$ at each vehicle $i$, based on all measurements (2)-(3) gathered by the interconnected vehicles. Relying on the diversity gain provided by the multiplicity of observations of the same passive features at different vehicles and on the $\mathrm{V} 2 \mathrm{~V}$ connectivity for fusion of such observations, the ICP approach is proposed in the following sections for improving vehicle positioning, based on both centralized (Sec. III) and distributed (Sec. IV) processing frameworks.

\section{Centralized ICP Method}

In this section, we describe the centralized approach for the joint estimation all vehicles/features' positions $\boldsymbol{\theta}_{t}=\left[\mathbf{x}_{t}^{\mathrm{T}}, \mathbf{f}^{\mathrm{T}}\right]^{\mathrm{T}}$ $\in \mathbb{R}^{\left(2 N_{v}+2 N_{f}\right) \times 1}$ that will be used as basis for the development of the distributed ICP method in Sec. IV.

Let $\mathbf{x}_{t}=\left[\mathbf{x}_{1, t}^{\mathrm{T}} \cdots \mathbf{x}_{N_{v}, t}^{\mathrm{T}}\right]^{\mathrm{T}} \in \mathbb{R}^{2 N_{v} \times 1}$ and $\mathbf{f}=$ $\left[\mathbf{f}_{1}^{\mathrm{T}} \cdots \mathbf{f}_{N_{f}}^{\mathrm{T}}\right]^{\mathrm{T}} \in \mathbb{R}^{2 N_{f} \times 1}$ be the vectors of, respectively, all vehicles' and features' positions at time $t$. The vector $\mathbf{y}_{t}^{(\mathrm{GPS})}=\left[\left(\mathbf{y}_{1, t}^{(\mathrm{GPS})}\right)^{\mathrm{T}} \cdots\left(\mathbf{y}_{N_{v}, t}^{(\mathrm{GPS})}\right)^{\mathrm{T}}\right]^{\mathrm{T}} \in \mathbb{R}^{2 N_{v} \times 1}$ collects all the GPS measurements (2) and $\mathbf{y}_{t}^{(\mathrm{V} 2 \mathrm{~F})} \triangleq\left[\mathbf{y}_{i, k, t}^{(\mathrm{V} 2 \mathrm{~F})}\right]_{i \in \mathcal{V}, k \in \mathcal{F}_{i, t}}=$ $\left[\left(\mathbf{y}_{1, t}^{(\mathrm{V} 2 \mathrm{~F})}\right)^{\mathrm{T}} \cdots\left(\mathbf{y}_{M, t}^{(\mathrm{V} 2 \mathrm{~F})}\right)^{\mathrm{T}}\right]^{\mathrm{T}} \in \mathbb{R}^{2 M \times 1}$ all the V2F observations (3). The V2F measurements are conveniently indexed as $\mathbf{y}_{m, t}^{(\mathrm{V} 2 \mathrm{~F})}=\mathbf{y}_{i_{m}, k_{m}, t}^{(\mathrm{V} 2 \mathrm{~F})}$, with $m=1, \ldots, M$ and $M=$ $\sum_{i=1}^{N_{v}}\left|\mathcal{F}_{i, t}\right|, m$ identifying the measurement $\left(i_{m}, k_{m}\right)$ made by the vehicle $i_{m} \in \mathcal{V}$ on the feature $k_{m} \in \mathcal{F}_{i_{m}, t}$.

In the centralized approach, measurements taken by all vehicles are aggregated by a fusion center into the vector:

$$
\mathbf{y}_{t}=\left[\begin{array}{l}
\mathbf{y}_{t}^{(\mathrm{GPS})} \\
\mathbf{y}_{t}^{(\mathrm{V} 2 \mathrm{~F})}
\end{array}\right]=\underbrace{\left[\begin{array}{cc}
\mathbf{I}_{2 N_{v}} & \mathbf{0}_{2 N_{v} \times 2 N_{f}} \\
\mathbf{M}_{v} & \mathbf{M}_{f}
\end{array}\right]}_{\mathbf{H}_{t}} \boldsymbol{\theta}_{t}+\underbrace{\left[\begin{array}{l}
\mathbf{n}_{t}^{(\mathrm{GPS})} \\
\mathbf{n}_{t}^{(\mathrm{V} 2 \mathrm{~F})}
\end{array}\right]}_{\mathbf{n}_{t}},
$$

and used to estimate the overall dynamic state $\boldsymbol{\theta}_{t} . \mathbf{H}_{t}$ is the known regressor matrix, where $\mathbf{M}_{v}=\left[\mathbf{M}_{m, i}\right]$ is blockpartitioned into $M \times N_{v}$ blocks of dimensions $2 \times 2$ defined as $\mathbf{M}_{m, i}=-\mathbf{I}_{2}$ if $i=i_{m}, \mathbf{M}_{m, i}=\mathbf{0}$ otherwise. Similarly, the matrix $\mathbf{M}_{f}=\left[\mathbf{M}_{m, k}\right]$, is block-partitioned into $M \times N_{f}$ blocks of dimensions $2 \times 2$ defined as $\mathbf{M}_{m, k}=\mathbf{I}_{2}$ if $k=k_{m}$, $\mathbf{M}_{m, k}=\mathbf{0}$ otherwise. The additive Gaussian measurement noise vector is $\mathbf{n}_{t} \sim \mathcal{N}\left(\mathbf{0}, \mathbf{R}_{t}\right)$, with $\mathbf{n}_{t}^{(\mathrm{GPS})}=\left[\mathbf{n}_{i, t}^{(\mathrm{GPS})}\right]_{i \in \mathcal{V}}$, $\mathbf{n}_{t}^{(\mathrm{V} 2 \mathrm{~F})}=\left[\mathbf{n}_{i, k, t}^{(\mathrm{V} 2 \mathrm{~F})}\right]_{i \in \mathcal{V}, k \in \mathcal{F}_{i, t}}$, and the noise covariance matrix is $\mathbf{R}_{t}=\operatorname{blockdiag}\left(\mathbf{R}_{1, t}^{(\mathrm{GPS})}, \ldots, \mathbf{R}_{N_{v}, t}^{(\mathrm{GPS})}, \mathbf{R}_{1, t}^{(\mathrm{V} 2 \mathrm{~F})}, \ldots, \mathbf{R}_{M, t}^{(\mathrm{V} 2 \mathrm{~F})}\right)$, with $\mathbf{R}_{m, t}^{(\mathrm{V} 2 \mathrm{~F})}=\mathbf{R}_{i_{m}, k_{m}, t}^{(\mathrm{V} 2 \mathrm{~F})}$ based on V2F measurement indexing.
The minimum mean square error (MMSE) estimate of $\boldsymbol{\theta}_{t}$ given all measurements $\mathbf{y}_{1: t}=\left\{\mathbf{y}_{1}, \ldots, \mathbf{y}_{t}\right\}$ up to time $t$ [11]

$$
\hat{\boldsymbol{\theta}}_{t \mid t}=\left[\begin{array}{c}
\hat{\mathbf{x}}_{t \mid t} \\
\hat{\mathbf{f}}_{t \mid t}
\end{array}\right]=\int \boldsymbol{\theta}_{t} p\left(\boldsymbol{\theta}_{t} \mid \mathbf{y}_{1: t}\right) d \boldsymbol{\theta}_{t},
$$

is extracted from the posterior probability density function (pdf), $p\left(\boldsymbol{\theta}_{t} \mid \mathbf{y}_{1: t}\right)$, which can be computed sequentially according to the Bayesian approach [11]:

$$
p\left(\boldsymbol{\theta}_{t} \mid \mathbf{y}_{1: t}\right) \propto p\left(\mathbf{y}_{t} \mid \boldsymbol{\theta}_{t}\right) \int p\left(\mathbf{x}_{t} \mid \mathbf{x}_{t-1}\right) p\left(\boldsymbol{\theta}_{t-1} \mid \mathbf{y}_{1: t-1}\right) d \mathbf{x}_{t-1},
$$

where $\propto$ stands for proportionality and the prediction has been simplified to account for the time-invariance of the features' positions. The likelihood function $p\left(\mathbf{y}_{t} \mid \boldsymbol{\theta}_{t}\right)=$ $p\left(\mathbf{y}_{t}^{(\mathrm{GPS})} \mid \mathbf{x}_{t}\right) p\left(\mathbf{y}_{t}^{(\mathrm{V} 2 \mathrm{~F})} \mid \boldsymbol{\theta}_{t}\right)$ can be calculated from the two independent measurement models (2)-(3) and the transition pdf $p\left(\mathbf{x}_{t} \mid \mathbf{x}_{t-1}\right)$ from the vehicle mobility model (1).

Recalling that all models are linear and Gaussian distributed, for the implementation of the centralized ICP localization (5)(6) we consider the Kalman filtering algorithm:

$$
\hat{\boldsymbol{\theta}}_{t \mid t}=\hat{\boldsymbol{\theta}}_{t \mid t-1}+\mathbf{C}_{t \mid t} \mathbf{H}_{t}^{T} \mathbf{R}_{t}^{-1}\left(\mathbf{y}_{t}-\mathbf{H}_{t} \hat{\boldsymbol{\theta}}_{t \mid t-1}\right),
$$

where $\hat{\boldsymbol{\theta}}_{t \mid t-1}$ is the prediction at time $t$ of all vehicles' states and features' locations:

$$
\hat{\boldsymbol{\theta}}_{t \mid t-1}=\left[\begin{array}{c}
\hat{\mathbf{x}}_{t \mid t-1} \\
\hat{\mathbf{f}}_{t \mid t-1}
\end{array}\right]=\left[\begin{array}{c}
\hat{\mathbf{x}}_{t-1 \mid t-1}+T_{s} \mathbf{v}_{t-1} \\
\hat{\mathbf{f}}_{t-1 \mid t-1}
\end{array}\right],
$$

with $\mathbf{v}_{t-1}=\left[\mathbf{v}_{i, t-1}\right]_{i \in \mathcal{V}}$ according to (1). The covariance of the estimate is:

$$
\mathbf{C}_{t \mid t}=\operatorname{Cov}\left(\hat{\boldsymbol{\theta}}_{t \mid t}\right)=\left(\mathbf{C}_{t \mid t-1}^{-1}+\mathbf{H}_{t}^{T} \mathbf{R}_{t}^{-1} \mathbf{H}_{t}\right)^{-1},
$$

where

$$
\mathbf{C}_{t \mid t-1}=\operatorname{Cov}\left(\hat{\boldsymbol{\theta}}_{t \mid t-1}\right)=\operatorname{blockdiag}\left(\mathbf{C}_{x, t \mid t-1}, \mathbf{C}_{f, t \mid t-1}\right),
$$

is the covariance of the prediction, with submatrix $\mathbf{C}_{x, t \mid t-1}=$ blockdiag $\left(\mathbf{C}_{x_{1}, t \mid t-1}, \ldots, \mathbf{C}_{x_{N_{v}}, t \mid t-1}\right)$ referring to the vehicles and $\mathbf{C}_{x_{i}, t \mid t-1}=\mathbf{C}_{x_{i}, t-1 \mid t-1}+\mathbf{Q}_{i, t-1}$ to vehicle $i \in \mathcal{V}$. The submatrix $\mathbf{C}_{f, t \mid t-1}=\operatorname{blockdiag}\left(\mathbf{C}_{f_{1}, t-1 \mid t-1}, \ldots, \mathbf{C}_{f_{N_{f}}, t-1 \mid t-1}\right)$ denotes the prior covariance of the features with $\mathbf{C}_{f_{k}, t \mid t-1}$ referring to feature $k \in \mathcal{F}$.

\section{Distributed ICP Method}

The centralized solution presented in the previous section is not feasible for large-scale networks as the performance is penalized by latency and communication/computational overhead required for data processing at the fusion center that badly scale with the network size. Furthermore, it is vulnerable to failure when fusion center breaks down. For these reasons, here we propose a distributed solution based on a combination of $\mathrm{BP}$ and consensus algorithms.

The distributed method enables the sequential evaluation, at each vehicle $i \in \mathcal{V}$, of the marginal posterior pdfs resulting from the factorization of (6), namely $p\left(\mathbf{x}_{i, t} \mid \mathbf{y}_{1: t}\right)$ and $p\left(\mathbf{f}_{k} \mid \mathbf{y}_{1: t}\right), \forall k \in \mathcal{F}$ (Sec. IV-A). However, as features are passive objects, they are not actively involved in the BP procedure. Thus, each vehicle $i$ has to calculate not only its 
own belief but also all features' beliefs using only V2V links. To address this challenge, we propose a new consensus-based BP method that allows cooperation between vehicles for the distributed evaluation of the features' beliefs (Sec. IV-B).

\section{A. Belief Propagation for V2V cooperation}

A BP method is designed to enable the evaluation at each vehicle of the posterior pdf of its own location and all features $k \in \mathcal{F}$. The message-passing approach is based on the Gaussian approximation and follows the algorithm in [12] here adapted to the specific localization problem. At time $t$, the marginal posterior pdfs are approximated at vehicle $i$ through iterations $n=1, \ldots, N_{\mathrm{bp}}$ by the beliefs:

$$
\begin{aligned}
& b_{i, t}^{(n)}\left(\mathbf{x}_{i, t}\right) \approx p^{(n)}\left(\mathbf{x}_{i, t} \mid \mathbf{y}_{1: t}\right), \\
& b_{k, t}^{(n)}\left(\mathbf{f}_{k}\right) \approx p^{(n)}\left(\mathbf{f}_{k} \mid \mathbf{y}_{1: t}\right) .
\end{aligned}
$$

Beliefs are initialized at BP step $n=0$ as:

$$
\begin{aligned}
& b_{i, t}^{(0)}\left(\mathbf{x}_{i, t}\right)=p\left(\mathbf{y}_{i, t}^{(\mathrm{GPS})} \mid \mathbf{x}_{i, t}\right) p\left(\mathbf{x}_{i, t} \mid \mathbf{y}_{1: t-1}\right), \\
& b_{k, t}^{(0)}\left(\mathbf{f}_{k}\right)=b_{k, t-1}^{\left(N_{\mathrm{bp}}\right)}\left(\mathbf{f}_{k}\right)
\end{aligned}
$$

where $b_{k, t-1}^{\left(N_{\mathrm{bp}}\right)}\left(\mathbf{f}_{k}\right)$ is the $k$ th feature belief at time slot $t-1$, while for the $i$ th vehicle $p\left(\mathbf{y}_{i, t}^{(\mathrm{GPS})} \mid \mathbf{x}_{i, t}\right)$ is the local GPS likelihood computed according to (2) and $p\left(\mathbf{x}_{i, t} \mid \mathbf{y}_{1: t-1}\right)=$ $\int p\left(\mathbf{x}_{i, t} \mid \mathbf{x}_{i, t-1}\right) b_{i, t-1}^{\left(N_{\mathrm{bp}}\right)}\left(\mathbf{x}_{i, t-1}\right) \mathrm{d} \mathbf{x}_{i, t-1}$ is the prior information, with $p\left(\mathbf{x}_{i, t} \mid \mathbf{x}_{i, t-1}\right)$ and $b_{i, t-1}^{\left(N_{\mathrm{bp}}\right)}\left(\mathbf{x}_{i, t-1}\right)$ respectively statetransition pdf from (1) and belief at time slot $t-1$.

At step $n>0$, the estimation procedure is carried out as summarized in the following, with the support of the illustrations in Fig. 2. The evaluation starts from the vehicle/feature beliefs available from step $n-1$ (see Fig. 2-(a)). In order to compute a belief about the feature- $k$ position, vehicle $i$ needs to combine the belief of its own location from step $n-1$ with the V2F likelihood $p\left(\mathbf{y}_{i, k, t}^{(\mathrm{V} 2 \mathrm{~F})} \mid \mathbf{f}_{k}, \mathbf{x}_{i, t}\right)$, evaluated according to the model (3). The resulting belief is provided as a message from vehicle $i$ to feature $k$ (dashed contours in Fig. 2-(a)):

$$
m_{i \rightarrow k}^{(n)}\left(\mathbf{f}_{k}\right) \propto \int p\left(\mathbf{y}_{i, k, t}^{(\mathrm{V} 2 \mathrm{~F})} \mid \mathbf{f}_{k}, \mathbf{x}_{i, t}\right) \frac{b_{i, t}^{(n-1)}\left(\mathbf{x}_{i, t}\right)}{m_{k \rightarrow i}^{(n-1)}\left(\mathbf{x}_{i, t}\right)} d \mathbf{x}_{i, t} .
$$

All messages on feature $k$ are then combined to extract a refined belief (black circle in Fig. 2-(b)):

$$
b_{k, t}^{(n)}\left(\mathbf{f}_{k}\right) \propto b_{k, t}^{(0)}\left(\mathbf{f}_{k}\right) \prod_{i \in \mathcal{V}_{k, t}} m_{i \rightarrow k}^{(n)}\left(\mathbf{f}_{k}\right),
$$

where $\mathcal{V}_{k, t}$ is the set of vehicles that acquire measurements of feature $k$. We assume that (16) is reset to a uniform distribution if the feature is not observed by any vehicle $i \in \mathcal{V}$. Based on the refined feature belief (16) and the V2F likelihood, vehicle $i$ receives a message from feature $k$ (see Fig. 2-(c)):

$$
m_{k \rightarrow i}^{(n)}\left(\mathbf{x}_{i, t}\right) \propto \int p\left(\mathbf{y}_{i, k, t}^{(\mathrm{V} 2 \mathrm{~F})} \mid \mathbf{f}_{k}, \mathbf{x}_{i, t}\right) \frac{b_{k, t}^{(n)}\left(\mathbf{f}_{k}\right)}{m_{i \rightarrow k}^{(n)}\left(\mathbf{f}_{k}\right)} d \mathbf{f}_{k} .
$$

The belief of vehicle $i \in \mathcal{V}$ is then updated by combining the vehicle's local information with all messages coming from all the features $k \in \mathcal{F}_{i, t}$ (colored ellipses in Fig. 2-(c)):

$$
b_{i, t}^{(n)}\left(\mathbf{x}_{i, t}\right) \propto b_{i, t}^{(0)}\left(\mathbf{x}_{i, t}\right) \prod_{k \in \mathcal{F}_{i, t}} m_{k \rightarrow i}^{(n)}\left(\mathbf{x}_{i, t}\right) .
$$

The BP steps (15)-(18) are repeated till convergence. Iterations stop when $\left\|\hat{\mathbf{f}}_{k, t}^{(n+1)}-\hat{\mathbf{f}}_{k, t}^{(n)}\right\|<\gamma_{\mathrm{mp}}, \forall k \in \mathcal{F}$, and $\| \hat{\mathbf{x}}_{i, t}^{(n+1)}-$ $\hat{\mathbf{x}}_{i, t}^{(n)} \|<\gamma_{\mathrm{mp}}, \forall i \in \mathcal{V}$, where $\gamma_{\mathrm{mp}}$ denotes a threshold, whereas $\hat{\mathbf{f}}_{k, t}^{(n)}$ and $\hat{\mathbf{x}}_{i, t}^{(n)}$ are the feature and vehicle location estimates at step $n$ based on the beliefs (16) and (18), respectively.

Note that since prior distributions of vehicles and features are Gaussian, models are linear in the state and have independent Gaussian noise, computations (14)-(18) reduce to the evaluation of linear combinations of mean vectors and covariances of beliefs/messages. The problem is the computation of the feature belief (16), as discussed in the following.

\section{B. Consensus Algorithm for Fusion of Features' Beliefs}

For the evaluation of (16), we need to compute the product of the messages from all vehicles sensing feature $k$ :

$$
u_{f_{k}}^{(n)}\left(\mathbf{f}_{k}\right) \triangleq \prod_{i \in \mathcal{V}_{k, t}} m_{i \rightarrow k}^{(n)}\left(\mathbf{f}_{k}\right)=\prod_{i \in \mathcal{V}} m_{i \rightarrow k}^{(n)}\left(\mathbf{f}_{k}\right),
$$

which can be conveniently expressed as a product over all the vehicles by extending the definition of the messages also to non-connected vehicle-feature pairs as follows: $m_{i \rightarrow k}^{(n)}\left(\mathbf{f}_{k}\right)$ is defined based on (15) if $i \in \mathcal{V}_{k, t}$, while for $i \notin \mathcal{V}_{k, t}$ it is set as a Gaussian pdf with covariance matrix tending to infinity.

Unfortunately, features are passive objects and thus they are not actively involved in the estimation process. A cooperation between vehicles through $\mathrm{V} 2 \mathrm{~V}$ communication links is required to merge the messages in (19). To solve the problem, we observe that the product of $N_{v}$ Gaussian pdfs in (19) is also a Gaussian function (though not normalized) [13]:

$$
u_{f_{k}}^{(n)}\left(\mathbf{f}_{k}\right)=\prod_{i=1}^{N_{v}} \underbrace{\mathcal{N}\left(\boldsymbol{\mu}_{i, k}, \mathbf{C}_{i, k}\right)}_{m_{i \rightarrow k}^{(n)}\left(\mathbf{f}_{k}\right)} \propto \mathcal{N}\left(\tilde{\boldsymbol{\mu}}_{k}, \tilde{\mathbf{C}}_{k}\right),
$$

with covariance $\tilde{\mathbf{C}}_{k}=\left(\sum_{i=1}^{N_{v}} \mathbf{C}_{i, k}^{-1}\right)^{-1}$ and mean $\tilde{\boldsymbol{\mu}}_{k}=$ $\tilde{\mathbf{C}}_{k} \cdot\left(\sum_{i=1}^{N_{v}} \mathbf{C}_{i, k}^{-1} \boldsymbol{\mu}_{i, k}\right)$, which can be expressed in terms of arithmetic average. Thus, for the cooperative evaluation of the first two moments of $u_{f_{k}}^{(n)}\left(\mathbf{f}_{k}\right)$ in (19), we propose to employ the average consensus approach [14], based on successive refinements of local estimates at vehicles and information exchange between neighbors. Namely, starting from the evaluation of the covariance matrix $\tilde{\mathbf{C}}_{k}$ and using for initialization $\boldsymbol{\Phi}_{i, k}^{(p)}=\mathbf{C}_{i, k}^{-1}$, the local estimate at vehicle $i$ is updated at iteration $p=1,2, \ldots$ as:

$$
\boldsymbol{\Phi}_{i, k}^{(p+1)}=\boldsymbol{\Phi}_{i, k}^{(p)}+\epsilon \sum_{j \in \mathcal{J}_{i, t}}\left(\boldsymbol{\Phi}_{j, k}^{(p)}-\boldsymbol{\Phi}_{i, k}^{(p)}\right) .
$$

based on the estimates $\boldsymbol{\Phi}_{j, k}^{(p)}$ provided by the neighbors $j \in \mathcal{J}_{i, t}$. For step-size $0<\epsilon<1 / \Delta_{t}$, the estimate is known to converge to the average of the initial estimates, i.e., $\boldsymbol{\Phi}_{i, k}^{(\infty)}=1 / N_{v} \sum_{i} \boldsymbol{\Phi}_{i, k}^{(0)}$ [14]. Thus, we easily obtain that $\tilde{\mathbf{C}}_{k}=\left(N_{v} \boldsymbol{\Phi}_{i, k}^{(\infty)}\right)^{-1}$. Similarly, for the cooperative evaluation of the mean $\tilde{\boldsymbol{\mu}}_{k}$, the consensus algorithm is initialized with $\boldsymbol{\Phi}_{i, k}^{(0)}=\mathbf{C}_{i, k}^{-1} \boldsymbol{\mu}_{i, k}$. Then, according to (21), we find that $\tilde{\boldsymbol{\mu}}_{k}=$ $N_{v} \tilde{\mathbf{C}}_{k} \boldsymbol{\Phi}_{i, k}^{(\infty)}$. In order to minimize communication costs, we 
(a)

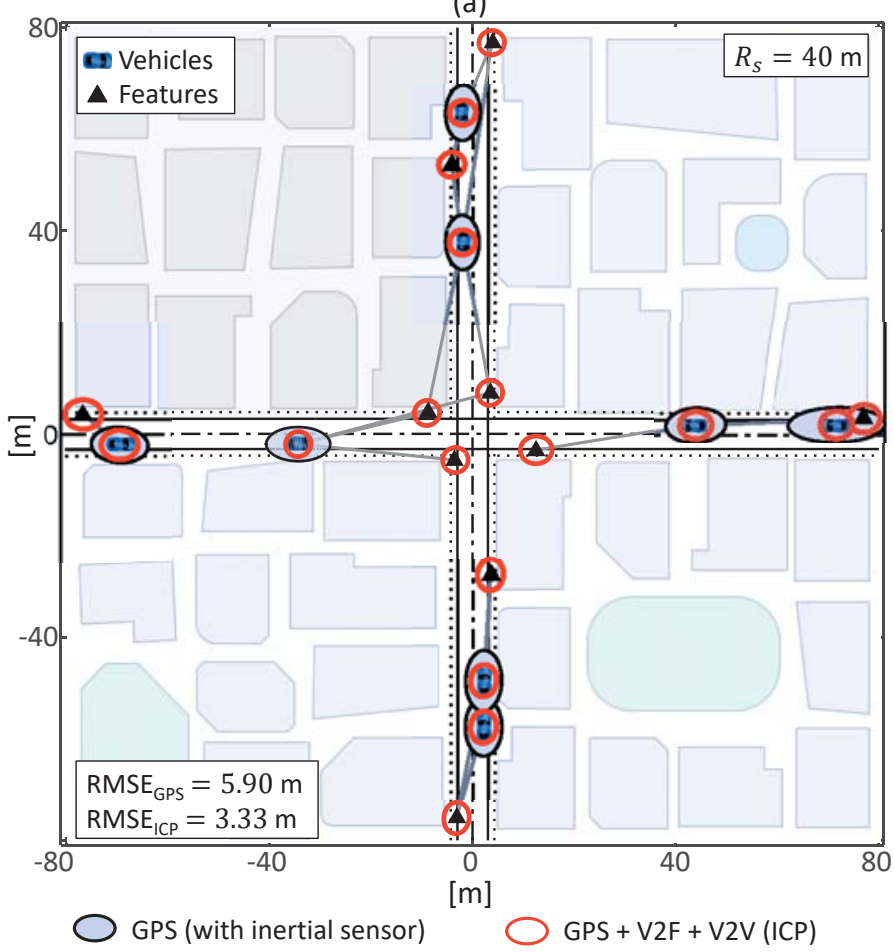

(b)

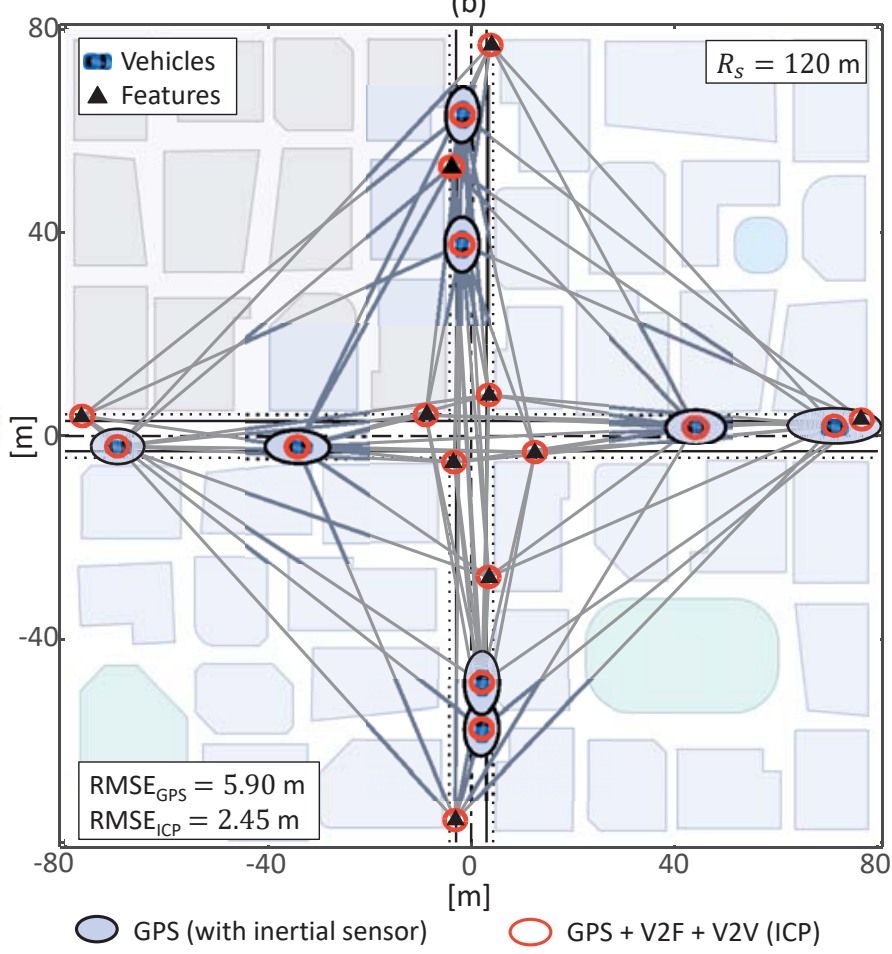

Fig. 3. Vehicle localization accuracy at time instant $t=50 \mathrm{~s}$ for sensing ranges (a) $R_{s}=40 \mathrm{~m}$ and (b) $R_{s}=120 \mathrm{~m}$ : stand-alone GPS method (light blue ellipse) and distributed ICP approach (red contour). RMSE values of vehicle position accuracy for all methods (bottom-left corner of the subfigures).

stop the consensus iterations when $\left\|\boldsymbol{\Phi}_{i, k}^{(p+1)}-\boldsymbol{\Phi}_{i, k}^{(p)}\right\|<\gamma_{\text {con }}$, $\forall i \in \mathcal{V}$ and $\forall k \in \mathcal{F}$. Once vehicles reach an agreement on $\tilde{\boldsymbol{\mu}}_{k}$ and $\tilde{\mathbf{C}}_{k}$, each vehicle can locally compute the approximate marginal posterior pdf of the feature $k$ and successively the message $m_{k \rightarrow i}^{(n)}\left(\mathbf{f}_{k}\right)$ according respectively to (16) and (17).

\section{Simulation Results}

We consider a $1.5 \mathrm{~km} \times 1.5 \mathrm{~km}$ urban canyon scenario, with two crossing roads as depicted in Fig. 1. Each road is a two-way two-lane street, with lane and sidewalk widths set to $3 \mathrm{~m}$ and $1.3 \mathrm{~m}$, respectively. The scenario involves $N_{v}$ vehicles, grouped in four clusters of $N_{v} / 4$ vehicles each, that enter at time $t=0$ from the four corners of the urban canyon area, with space headway $24 \mathrm{~m}$, drive straight ahead along their respective lanes at average speed of $50 \mathrm{~km} / \mathrm{h}$, cross the intersection and exit on the opposite sides. Each vehicle's motion is simulated according to (1), with driving process covariance $\mathbf{Q}_{i, t}=\operatorname{diag}\left(\sigma_{v_{x i}}^{2}, \sigma_{v_{y i}}^{2}\right)$. The velocity uncertainty in the direction of road is $\sigma_{v_{\|}}=2 \mathrm{~m} / \mathrm{s}$, while the one in the orthogonal direction is $\sigma_{v_{\perp}}=0.01 \mathrm{~m} / \mathrm{s}$; depending on the driving direction of the vehicle, the velocity uncertainties along axes $x\left(\sigma_{v_{x i}}\right)$ and $y\left(\sigma_{v_{y i}}\right)$ are defined accordingly. The sampling time is set to $T_{s}=1 \mathrm{~s}$. The $N_{f}$ features are randomly deployed along the sidewalks of the two roads, in an area of dimensions $700 \mathrm{~m} \times 700 \mathrm{~m}$ centered around the crossroad.

For positioning, vehicles rely solely on GPS - integrated with the on-board inertial sensor by Kalman filtering - until they start sensing some common features and they can benefit from V2V cooperation by the ICP method. The GPS measurement covariance matrix at each vehicle is $\mathbf{R}_{i, t}^{(\mathrm{GPS})}=\sigma_{\mathrm{GPS}}^{2} \mathbf{I}_{2}$, with $\sigma_{\mathrm{GPS}}=20 \mathrm{~m}$. The covariance matrix of $\mathrm{V} 2 \mathrm{~F}$ sensing is $\mathbf{R}_{i, k, t}^{(\mathrm{V} 2 \mathrm{~F})}=\sigma_{\mathrm{V} 2 \mathrm{~F}}^{2} \mathbf{I}_{2}$, with $\sigma_{\mathrm{V} 2 \mathrm{~F}}=0.1 \mathrm{~m}$ (as representative of radar accuracy). The communication range at each vehicle is set to $R_{c}=200 \mathrm{~m}$, while the sensing range $R_{s}$ is assumed to be lower and varies through simulations. The consensus step-size is set to $\epsilon=0.99 / \Delta_{t}$, while thresholds for $\mathrm{BP}$ and consensus convergence are set to $\gamma_{\mathrm{bp}}=\gamma_{\mathrm{con}}=10^{-3}$. Note that faster consensus methods can be used (e.g., with Metropolis weights), but these are not evaluated in this paper.

The positioning performance is evaluated through Monte Carlo simulations in terms of root mean square error (RMSE) of the vehicle position estimate over the area covered by features, where the ICP algorithm can be employed. An example of simulation is in Fig. 3, where a zoomed view of the urban canyon area is shown with $N_{v}=8$ vehicles and $N_{f}=8$ features in proximity of the intersection. Fig. 3 presents the average performance at time instant $t=50 \mathrm{~s}$, based on the $2 \times 2$ mean square error matrix of vehicles and features' position estimates at convergence, respectively $\mathbf{M S E}_{i}=E\left[\left(\hat{\mathbf{x}}_{i, t}-\mathbf{x}_{i, t}\right)\left(\hat{\mathbf{x}}_{i, t}-\mathbf{x}_{i, t}\right)^{T}\right]$ and $\mathbf{M S E} \mathbf{E}_{k}=E\left[\left(\hat{\mathbf{f}}_{k, t}-\right.\right.$ $\left.\left.\mathbf{f}_{k}\right)\left(\hat{\mathbf{f}}_{k, t}-\mathbf{f}_{k}\right)^{T}\right]$. Averages have been taken over 400 independent observations, for both the stand-alone GPS (with inertial sensor) and the distributed ICP algorithms. The error ellipses, at $50 \%$ confidence, are plotted around the vehicle (blue car) and feature (black triangle) positions. In particular, Figs. 3-(a) and (b) show the performances of the distributed ICP method (red contour) for sensing ranges $R_{s} \in\{40,120\}$ m compared to the conventional GPS solution (light blue ellipse). The V2F connectivity is also given (grey lines). Results show that the proposed distributed ICP method outperforms the stand-alone 


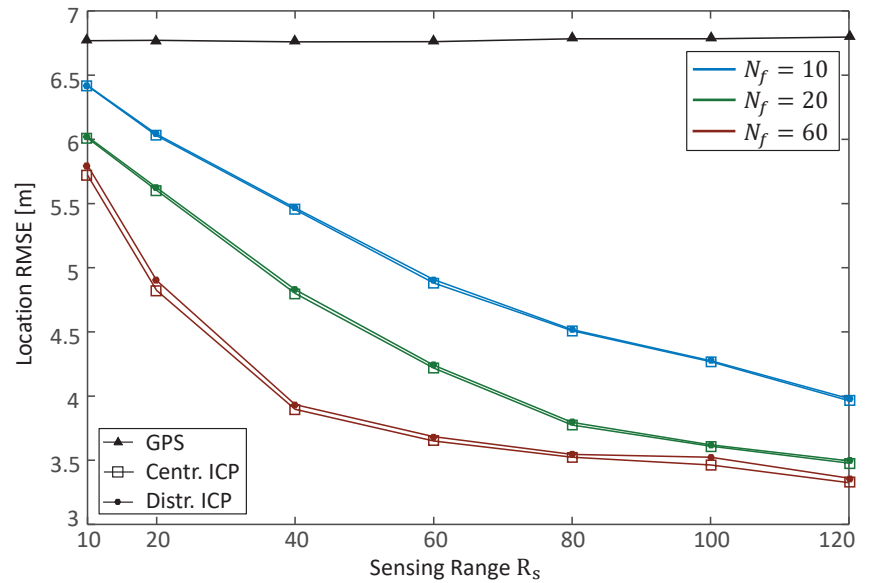

Fig. 4. RMSE of the vehicles' location estimates vs. different sensing ranges and for $N_{f} \in\{10,20,60\}$ features. Performance of the distributed ICP approach is compared with the stand-alone GPS and centralized ICP methods.

GPS, as confirmed by the comparison in terms of RMSE of the vehicle position estimate, averaged over all vehicles, on the bottom-left corner of the figures.

In Fig. 4, we consider a scenario with $N_{v}=12$ vehicles, $N_{f} \in\{10,20,60\}$ features and sensing range varying from $R_{s}=10 \mathrm{~m}$ up to $R_{s}=120 \mathrm{~m}$. The position RMSE, averaged over vehicles and time, is plotted for the three methods, namely the stand-alone GPS, the centralized and distributed versions of the proposed ICP method. The accuracy of the ICP algorithm increases with the sensing range and the availability of features. The distributed implementation is shown to closely attain the centralized approach. The ICP algorithm reduces the GPS error from $7 \mathrm{~m}$ down to $3.5 \mathrm{~m}$.

A more in depth analysis of the performance is in Fig. 5, which shows the cumulative density function (CDF) of the ICP localization error, for sensing range $R_{s}=40 \mathrm{~m}$ and $R_{s}=120$ m (dashed and solid lines), for $N_{f} \in\{10,20,60\}$ features. The proposed ICP method is compared to the stand-alone GPS (black dashed-dot line). For sensing range $R_{s}=40 \mathrm{~m}$ and $50 \%$ of confidence level, the distributed ICP approach achieves a location accuracy of $5.25 \mathrm{~m}, 4.54 \mathrm{~m}$ and $3.45 \mathrm{~m}$ respectively for 10, 20 and 60 features, while the stand-alone GPS accuracy is $6.64 \mathrm{~m}$. Moreover, when the V2F connectivity range is large enough (e.g., $R_{s}=120 \mathrm{~m}$ ), the proposed method can reach a good accuracy of $3 \mathrm{~m}$ with only 20 features.

\section{CONCLUSION}

In this paper, a novel cooperative positioning method in vehicular networks was proposed exploiting non-cooperative features as common noisy reference points that were localized by vehicles and implicitly used to enhance the GPS vehicle accuracy. To solve the positioning problem, we developed a consensus-based BP algorithm, where a consensus method was nested within the BP algorithm to enable cooperation between vehicles for the distributed estimation of the features' positions. The proposed ICP method was shown to closely attain the equivalent centralized approach and to provide meaningful performance gains in terms of vehicle position accuracy with respect to the stand-alone GPS solution.

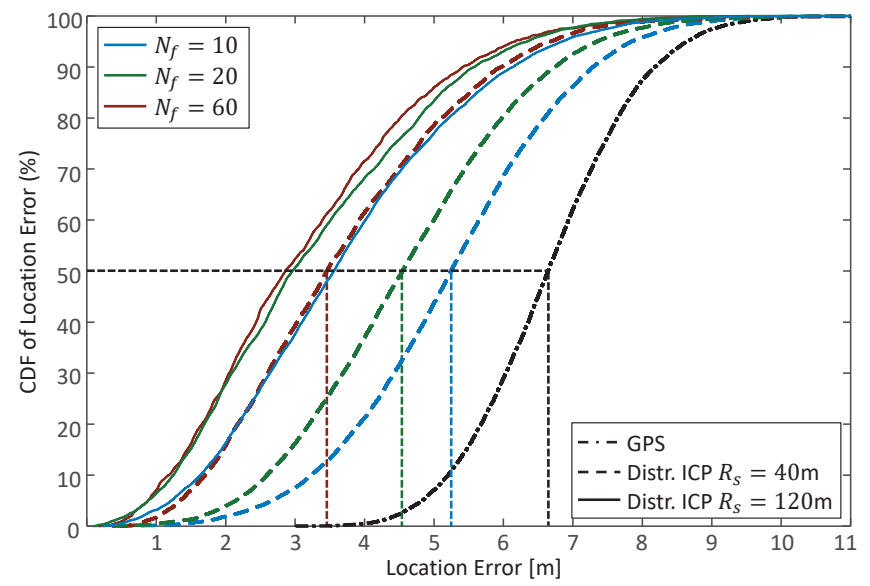

Fig. 5. CDF of the vehicles' location error. Performance results of the distributed ICP algorithm for $R_{s}=40 \mathrm{~m}$ and $R_{s}=120 \mathrm{~m}$ and for $N_{f} \in\{10,20,60\}$ are compared with the stand-alone GPS approach.

\section{ACKNOWLEDGEMENT}

This work was supported by COPPLAR, funded under Strategic Vehicle Research and Innovation Grant No. 2015-04849, by the H2020 project HIGHTS MG-3.5a2014-636537 and by Cluster Tecnologico Nazionale MIE CTN01_00034_594122 funded by MIUR.

\section{REFERENCES}

[1] X. Li, W. Shu, M. Li, H.-Y. Huang, P.-E. Luo, and M.-Y. Wu, "Performance Evaluation of Vehicle-based Mobile Sensor Networks for Traffic Monitoring," IEEE Tran. on Vehicular Technology, vol. 58, no. 4, pp. 1647-1653, 2009

[2] E. Kaplan and C. Hegarty, Understanding GPS: Principles and Applications. Norwood: Artech House, 2006.

[3] S. E. Shladover and S.-K. Tan, "Analysis of Vehicle Positioning Accuracy Requirements for Communication-based Cooperative Collision Warning," J. of Intelligent Transportation Systems, vol. 10, no. 3, pp. 131-140, 2006.

[4] J. B. Kenney, "Dedicated Short-Range Communications (DSRC) Standards in the United States," Proc. of the IEEE, vol. 99, no. 7, pp. 1162 1182, Jul. 2011.

[5] H. Wymeersch, J. Lien, and M. Z. Win, "Cooperative Localization in Wireless Networks," Proc. of the IEEE, vol. 97, no. 2, pp. 427-450, Feb. 2009.

[6] G. Soatti, M. Nicoli, S. Savazzi, and U. Spagnolini, "Consensus-based Algorithms for Distributed Network-State Estimation and Localization," IEEE Tran. on Signal and Inf. Processing over Networks, Jun. 2017.

[7] R. Parker and S. Valaee, "Vehicular Node Localization using ReceivedSignal-Strength Indicator," IEEE Tran. on Vehicular Technology, vol. 56, no. 6, pp. 3371-3380, 2007.

[8] M. Rohani, D. Gingras, and D. Gruyer, "Vehicular Cooperative Map Matching," in IEEE ICCVE, 2014, pp. 799-803.

[9] N. Alam and A. G. Dempster, "Cooperative Positioning for Vehicular Networks: Facts and Future," IEEE Tran. on Intelligent Transportation Systems, vol. 14, no. 4, pp. 1708-1717, Dec. 2013.

[10] N. M. Drawil and O. Basir, "Intervehicle-Communication-Assisted Localization," IEEE Tran. on Intelligent Transportation Systems, vol. 11, no. 3, pp. 678-691, 2010.

[11] S. M. Kay, Fundamentals of Statistical Signal Processing, volume I: Estimation Theory. Prentice Hall, 1993.

[12] J. Pearl, Probabilistic reasoning in intelligent systems. Morgan Kaufmann, 1988.

[13] F. R. Kschischang, B. J. Frey, and H.-A. Loeliger, "Factor Graphs and the Sum-Product Algorithm," IEEE Tran. on Information Theory, vol. 47, no. 2, pp. 498-519, 2001.

[14] R. Olfati-Saber, J. A. Fax, and R. M. Murray, "Consensus and Cooperation in Networked Multi-Agent Systems," Proc. of the IEEE, vol. 95, no. 1, pp. 215-233, 2007. 